\title{
Molecular Motion of Polymers in Concentrated Solutions
}

\author{
Michio KuRATA \\ Institute for Chemical Research, Kyoto University, \\ Uji, Kyoto 611, Japan
}

(Received August 20, 1984)

\begin{abstract}
The molecular motion of polymers in concentrated solutions is studied on the basis of recent measurements of relaxation stresses in the nonlinear region of viscoelasticity. The double-step deformation with varying time interval is proved to be specifically effective for studying not only a slow mode of the motion known as the reptation, but also a faster mode often referred to as the wriggling motion. It is found that in the former mode, the entanglement points behave as slip-links as assumed in the Doi-Edwards theory, while in the latter mode, they turn to be nonslip-links, as assumed in the recent Doi theory. The transfer of monomers across the entanglement points is practically inhibited at least in the early stage of the wriggling motion. The concept of non-slippery entanglements corresponds to the independent alignment approximation (IAA) of Doi and Edwards, which leads to the rheological constitutive model of Bernstein, Kearsley, and Zapas (BKZ model).
\end{abstract}

KEY WORDS Concentrated Polymer Solution / Nonlinear Viscoelasticity / Double-Step Deformation / Doi-Edwards' Theory / Polymer Entanglement / Rheological Constitutive Equation /

$\mathrm{We}^{1}$ found in 1971 that the relaxation modulus of a concentrated polystyrene solution in a single-step shear strain was factored into two parts, one depending on time $t$ and the other on strain $\gamma$, at times longer than a certain value $\tau_{k}$ :

$$
G(t, \gamma)=h(\gamma) G(t) \quad t>\tau_{k}
$$

This form of the strain-dependent modulus $G(t, \gamma)$ was compatible with a rheological constitutive model due to Bernstein, Kearsley, and Zapas (BKZ model). ${ }^{2}$

Five years later, the wide applicability of the BKZ model was established by systematic measurements of a variety of flow properties, which includes the steady shear viscosity as a function of shear rate, the stress development following sudden start of shear flow, the stress decay following cessation of shear flow, and the stress relaxation following application of double-step strain. ${ }^{3,4}$ At the same time, a failure of the BKZ model was also revealed in the case of double-step strain with antiparallel directions. ${ }^{5}$

The molecular dynamics underlying the above results was discovered by Doi and Edwards ${ }^{6}$ in 1978. Based on the primitive chain model, they have given a universal expression for the shift factor $h(\gamma)$ in eq 1 , which is in excellent agreement with the observed values. They have also shown that the theory, if combined with the so-called independent alignment approximation (IAA), yields the BKZ constitutive equation, without spoiling the gain concerned with $h(\gamma)$. Furthermore, Doi $^{7}$ has shown later that the theory gives a correct answer to the relaxation stress after application of antiparallel double-step strain with a long time interval, to which the BKZ model, hence IAA, produced a considerable error.

Reading the above progress of studies, one might think that the Doi-Edwards theory has two versions, i.e., the rigorous version without IAA and the approximate version with IAA. However, this is misleading. As has been noted in the Doi-Edwards article ${ }^{6}$ these two versions are simply based on two plausible assumptions on the slipperiness of entanglement points, so that there is no a priori reason for discriminating two versions by the terms, rigorous and approximate. In other words, the former version without IAA corresponds to the perfect slippage, while the latter with IAA to the non- 
slippage of entanglement points. Thus, evaluation of IAA which is essential for a clear understanding of the wriggling motion of polymers has to be made on the experimental basis.

We have recently pointed out elsewhere ${ }^{8}$ that in double-step shear involving the reversal of shearing direction, the IAA is successful for rapid reversal as compared with the time scale $\tau_{k}$ in eq 1 , though it fails for slower reversal. The situation is somewhat complicated. Thus, I find it worthwhile to review our efforts devoted to evaluation of IAA.

\section{STRESS RELAXATION IN SINGLE-STEP SHEAR}

Typical data of the relaxation modulus obtained for a polystyrene solution ${ }^{9}$ are shown in Figure 1, where the curve at the top represents the linear relaxation modulus $G(t)$ for sufficiently small strains, $\gamma<0.6$, and the remaining curves represent the strain-dependent moduli $G(t, \gamma)$ for larger strains. The long-time portion of these curves have the same shape so that they can be superposed on the $G(t)$-curve by vertical shift alone. The result is shown in Figure 2. In this way, we can determine the strain function $h(\gamma)$ in eq 1 , and two time constants, $\tau_{1}$ and $\tau_{k}$, as indicated by the arrows. The $\tau_{1}$ represents the longest relaxation time of the test

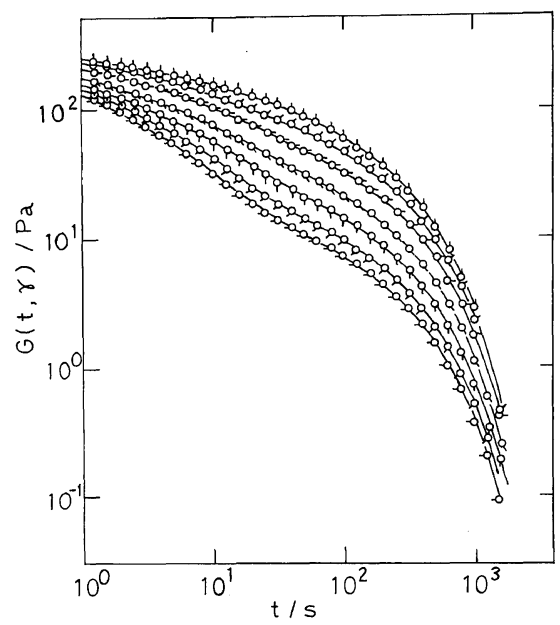

Figure 1. Relaxation modulus $G(t, \gamma)$ for a polystyrene solution in chlorinated biphenyl at $30^{\circ} \mathrm{C}$. Polymer molecular weight $M_{w}$ is $8.42 \times 10^{6}$ and concentration $c$ is $0.06 \mathrm{~g} \mathrm{~cm}^{-3}$. Amounts of shear $\gamma$ are $<0.57,1.25,2.06$, $3.04,4.0,5.3$, and 6.1 from top to bottom. system.

The values of $h(\gamma)$ obtained for various sytems are shown in Figure 3. Obviously, the $h(\gamma)$ is a universal function independent of polymer molecular weight $M$ and concentration $c$.

The linear relaxation modulus $G(t)$ is a material function. The ratio $\tau_{1} / \tau_{k}$ for various combinations of $M$ and $c$ is plotted against the number of entanglements per molecule, $M / M_{\mathrm{e}}$, in Figure $4 .^{10}$ Here the entanglement spacing $M_{\mathrm{e}}$ was evaluated from the relation

$$
c M_{\mathrm{e}}=2 \times 10^{4} \mathrm{~g} \mathrm{~cm}^{-3} \quad c \geqq 0.3 \mathrm{~g} \mathrm{~cm}^{-3}
$$

which is applicable for polystyrene solutions. The

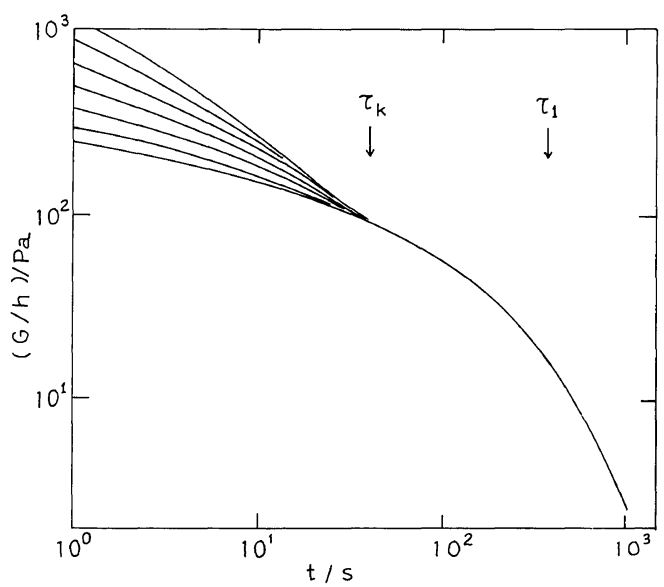

Figure 2. Reduced relaxation modulus $G(t, \gamma) / h(\gamma)$ derived from Figure 1.

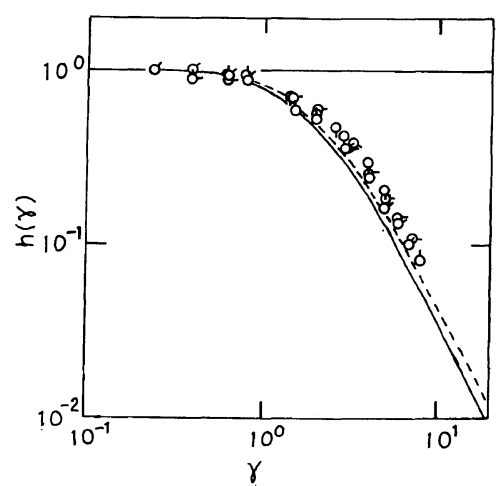

Figure 3. Shift factor $h(\gamma)$ for polystyrene solutions in chlorinated biphenyl. The circles with pip up, $M=$ $9.5 \times 10^{5}, c=0.30 \mathrm{~g} \mathrm{~cm}^{-3}$; succesive $45^{\circ}$-clockwise rotations of pip represent $M=9.5 \times 10^{5}, c=0.20 \mathrm{~g} \mathrm{~cm}^{-3}$; $M=6.7 \times 10^{5}, c=0.30 \mathrm{~g} \mathrm{~cm}^{-3}$; and $M=2.3 \times 10^{5}, c=$ $0.40 \mathrm{~g} \mathrm{~cm}^{-3}$, respectively. 


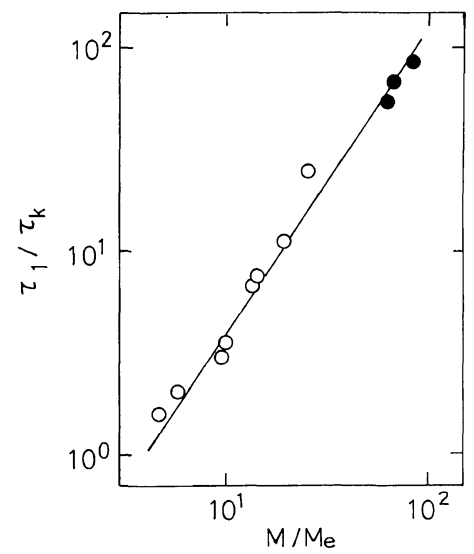

Figure 4. $\tau_{1} / \tau_{\mathrm{k}}$ plotted against $M / M_{\mathrm{e}}$, the number of entanglements per molecule, for polystyrene solutions. Filled circles are for polystyrenes with $M=5.6 \times 10^{6}$ and $7.6 \times 10^{6}$ in diethyl phthalate, and unfilled circles are for polystyrenes with $M=2.3 \times 10^{5}, 6.7 \times 10^{5}, 9.5 \times 10^{5}$, and $1.80 \times 10^{6}$ in chlorinated biphenyl. Slope of line is 1.5 .

line in Figure 4 represents

$$
\tau_{1} / \tau_{k} \propto\left(M / M_{\mathrm{e}}\right)^{1.5}
$$

Since $\tau_{1} \propto M^{3.5}$, we obtain

$$
\tau_{k} \propto\left(M / M_{\mathrm{e}}\right)^{2}
$$

The behavior of $G(t, \gamma)$ outlined above indicates that the stress relaxation after application of singlestep strain is divided in two processes by the time constant $\tau_{k}$.

\section{DOI-EDWARDS SLIP-LINK MODEL}

In the Doi-Edwards theory, ${ }^{6}$ each polymer in a highly entangled system is represented by a primitive chain which moves along the center path of a tube-like region. At equilibrium, the primitive chain assumes a random walk configuration of $N$ steps, each having length $a$ and representing a part of polymer chain divided by an adjacent pair of entanglement points. Thus, the number of primitive steps $N$ is equated to the number of entanglements per molecule. If $b$ is the length of a Rouse segment and $N_{0}$ is its number per molecule, the equilibrium contour length $L_{\mathrm{eq}}$ of the primitive chain is written as

$$
L_{\text {eq }}=N a=N_{0} b^{2} / a
$$

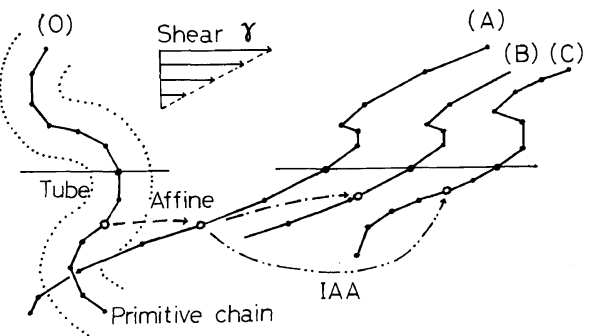

Figure 5. Change in the form of a primitive chain. (O), The original state in equilibrium; (A), the affinely deformed state; (B), the equilibrated state at the end of process $B$ due to the slip-link model; $(\mathrm{C})$, the equilibrated state at the end of process $B$ due to the independent alignment approximation (IAA). The dotted line represents the undeformed tube.

step is written as

$$
N_{\mathrm{e}}=N_{0} / N=a^{2} / b^{2}
$$

On application of a stepwise shear strain to the system, it is assumed that the tube, together with the primitive chain, is deformed affinely in accord with the macroscopic deformation. Each primitive step is extended or compressed and oriented according to its position relative to the deformation gradient as illustrated by the solid lines $\mathrm{O}$ and $\mathrm{A}$ in Figure 5.

The extended contour length $L$ and the heterogenized step-length $a$ or segment-density $N_{\mathrm{e}} / a$ in the chain $\mathrm{A}$ are equilibrated by wriggling motion of the Rouse chain in the equilibration time $T_{\text {eq }}$ of de Gennes. ${ }^{11}$ A considerable part of induced stress is relaxed in this process $\mathrm{B},{ }^{12}$ which determines the strain function $h(\gamma)$ in eq 1 .

Doi and Edwards have calculated $h(\gamma)$ by two methods. In the first method, the extended contour length of the chain $A$ is assumed to return to the equilibrium value $L_{\mathrm{eq}}$ along the center path of the tube as illustrated by the line B in Figure 5. Due to the contraction of the contour length, the number of primitive steps decreases, while the number of Rouse segments in each survived step increases. Counting the balance of these effects, Doi and Edwards have derived the following expression for the stress tensor in the state $\mathrm{B}$ :

$$
\begin{gathered}
\sigma_{\alpha \beta}(B)=\rho N k_{\mathrm{B}} T Q_{\alpha \beta}(E) \quad\left(t \cong T_{\mathrm{eq}}\right) \\
(\alpha, \beta=1,2,3)
\end{gathered}
$$

and the number $N_{\mathrm{e}}$ of Rouse segments per primitive 


$$
Q_{\alpha \beta}(\boldsymbol{E})=\frac{1}{\langle|\boldsymbol{E} \cdot \boldsymbol{u}|\rangle}\left\langle\frac{(\boldsymbol{E} \cdot \boldsymbol{u})_{\alpha}(\boldsymbol{E} \cdot \boldsymbol{u})_{\beta}}{|\boldsymbol{E} \cdot \boldsymbol{u}|}\right\rangle(\alpha, \beta=1,2,3)
$$

where $\rho$ is the number of polymers in unit volume, $k_{\mathrm{B}}$ the Boltzmann constant, $T$ the absolute temperature, $\boldsymbol{u}$ the unit vector along the primitive chain, $\boldsymbol{E}$ the transpose of the deformation gradient tensor, and $\langle\cdots\rangle$ the isotropic average of the contents. In this process from state $\mathbf{A}$ to $\mathbf{B}$, Rouse segments are assumed to move freely across entanglement points, just like across slip-links under a hypothetical force $F_{\text {eq }}=3 k T / a$. As a result a Rouse segment located on an entanglement point is transferred to an intermediate site on a primitive step as indicated by the unfilled circles.

The second method is IAA, in which the modified length of each step of the chain $\mathrm{A}$ is assumed to return individually to the equilibrium value $a$ as illustrated by the line $\mathrm{C}$. Thus, it demands no change in the number of steps per molecule nor in the number of Rouse segments per step, but a local adjustment of the primitive path, and finally it gives

$$
Q_{\alpha \beta}(\boldsymbol{E})=\left\langle\frac{(\boldsymbol{E} \cdot \boldsymbol{u})_{\alpha}(\boldsymbol{E} \cdot \boldsymbol{u})_{\beta}}{|\boldsymbol{E} \cdot \boldsymbol{u}|^{2}}\right\rangle
$$

In this process from state $\mathrm{A}$ to $\mathrm{C}$, Rouse segments do not move across entanglement points. In other words, entanglements act as non-slip-links.

The function $h(\gamma)$ in eq 1 can be calculated from $Q_{12}(\gamma)$ as

$$
h(\gamma)=\left[\gamma^{-1} Q_{12}(\gamma)\right] / \lim _{\gamma \rightarrow 0}\left[\gamma^{-1} Q_{12}(\gamma)\right]
$$

The results obtained by eq 8 and 9 are shown by solid and dashed lines in Figure 3, respectively. They are practically indistinguishable and in good agreement with experimental data.

The anisotropic orientation of the primitive chain in state $\mathrm{B}$ or $\mathrm{C}$ is equilibrated as the chain is disengaged by reptation from the original tube. This process $\mathrm{C}$ in Doi's terminology ${ }^{12}$ is characterized by the disengagement time $T_{\mathrm{d}}$ of de Gennes. ${ }^{11}$ Since the disengaged part of the chain are isotropic, the orientation angle is determined solely by the middle part of the chain remaining in the original tube and is kept constant until the middle part vanishes. A schematic diagram of this process may be found in the recent edition of Ferry's book. ${ }^{13}$

The equilibration time $T_{\text {eq }}$ and the disengagement time $T_{\mathrm{d}}$ are given as

$$
\begin{gathered}
T_{\text {eq }}=2\left(M / M_{\mathrm{e}}\right)^{2} \tau_{\mathrm{A}} \\
T_{\mathrm{d}}=6\left(M / M_{\mathrm{e}}\right)^{3} \tau_{\mathrm{A}}
\end{gathered}
$$

where $\tau_{\mathrm{A}}$ represents the longest relaxation time of a Rouse chain with molecular weight $M_{\mathrm{e}}$. Comparison of eq 11 with eq 4 indicates that $T_{\text {eq }}$ is proportional to $\tau_{k}$. Here we put ${ }^{3}$

$$
T_{\text {eq }}=\tau_{k} / 4
$$

Equation 12 does not agree with the familiar 3.5th power dependence of $T_{\mathrm{d}}$ on $M$. In this article, we are not concerned with this discrepancy, but simply note that Doi has recently presented a possible solution. ${ }^{14}$

\section{ORIENTATION OF POLYMERS IN SINGLE-STEP SHEAR}

The single-step shear deformation may be represented by a displacement vector $d$, whose components are $d_{1}=d_{2}=d_{3}=0$ for $t<0$, and $d_{1}=\gamma x_{2}$, $d_{2}=d_{3}=0$ for $t \geqq 0$, with respect to a Cartesian coordinate system $x_{1} x_{2} x_{3}$. Then, for an incompressible material, the rheologically signigicant stresses are the shear stress $\sigma_{12}$, the first normal stress difference $\sigma_{11}-\sigma_{22}$, and the second normal stress difference $\sigma_{22}-\sigma_{33}$ or its alternative $\sigma_{11}-\sigma_{33}$. For simplicity, we denote these stress components by $\sigma, v$, and $v_{2}$ or $v_{3}$, respectively. The stress-optical law is then expressed as

$$
\begin{gathered}
2 \sigma=(\Delta n / C) \sin 2 \chi \\
v=(\Delta n / C) \cos 2 \chi \\
v_{3}=v+v_{2}=\left(\Delta n_{13} / C\right)
\end{gathered}
$$

where $\Delta n$ is the birefringence in the $x_{1} x_{2}$-plane, $\Delta n_{13}$ is that in the $x_{1} x_{3}$-plane, $\chi$ is the extinction angle, and $C$ is the stress-optical coefficient.

Figure 6 shows typical results of optical and shear stress measurements in the time-dependent field after the single-step shear. ${ }^{15}$ The solution is a polystyrene in polychlorinated biphenyl (Aroclor 1248), with $M_{w}=6.7 \times 10^{5}$ and $c=0.40 \mathrm{~g} \mathrm{~cm}^{-3}$. In the figure, values of quantities, $|\Delta n|,(2 \cot 2 \chi) / \gamma$, and $|C|=(|\Delta n| \sin 2 \chi) / 2 \sigma$, measured at various $\gamma$ are plotted against time. The extinction angle $\chi$ was independent of time $t$, and the quantity $|C|$ was also independent of $t$, indicating the validity of the stress optical law. ${ }^{15}$ Furthermore, we found 


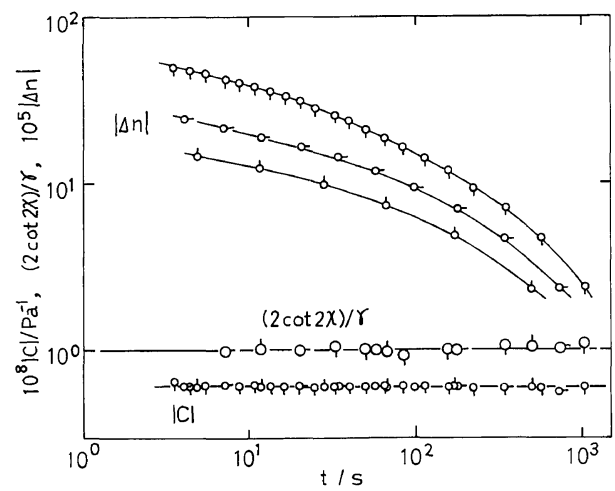

Figure 6. Quantities $|\Delta n|,(2 \cot 2 \chi) / \gamma$, and $|C|=$ $(|\Delta n| \sin 2 \chi) / 2 \sigma$ plotted against time $t$ for three values of shear strain $\gamma$ : circles with pip up, 0.68 ; pip right, 1.01; pip down, 2.07. The test solution is described in the text.

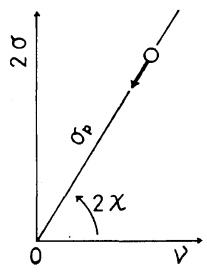

Figure 7. Two dimensional diagram of the stress state $(\gamma, 2 \sigma)$ after application of a single-step strain. The polar coordinates of the circle are given by $\sigma_{\mathrm{P}}(t)$ and $2 \chi$, the latter is invariant in the relaxation process.

$$
\cot 2 \chi=\nu / 2 \sigma=\gamma / 2
$$

The last equality is called the Lodge-Meissner relation.

Thus, if $2 \sigma(t, \gamma)$ is plotted against $v(t, \gamma)$, we obtain a straight line as illustrated in Figure 7 . The angle of the line with the $v$ axis is equal to $2 \chi$, and the point representing the instantaneous stress state moves on the line towards the origin as $t$ increases. The radial distance $\sigma_{\mathrm{P}}$ is $\Delta n / C$. The $\sigma_{\mathrm{P}}$ gives a measure of the tensile force acting along the extended primitive chain in the relaxation process $\mathrm{B}$, and a measure of the amount of Rouse segments confined in the original tube in the process $C$ where the tensile force is already equilibrated. This diagram is useful for monitoring the change in anisotropic orientation of polymers.

The ratio $v_{2} / v$, which was negative, also did not depend on time $t$, and its dependence on $\gamma$ obeyed approximately the theoretical function given by Doi and Edwards, though not reproduced here. ${ }^{15,17}$

\section{STRESS RELAXATION IN DOUBLE-STEP SHEAR}

Now we proceed to a double-step shear deformation such that a stepwise shear of magnitude $\gamma_{1}$, either positive or negative, is applied at time $-t_{3}$ and another shear $\gamma_{2}$, always positive, is added at $t=0$. The shear stress $\sigma$ and the normal stress difference $v_{3}$ are measured for $t>0$, under various combinations of $t_{1}, \gamma_{1}$, and $\gamma_{2}$. Thus, we write the measured stresses as $\sigma\left(t, t_{1}, \gamma_{1}, \gamma_{2}\right)$ and $v_{3}\left(t, t_{1}, \gamma_{1}\right.$, $\left.\gamma_{2}\right)$. We did not measure $v_{2}$ directly because of experimental difficulty, but estimated it approximately by

$$
v_{2}=-0.2 v_{3} \quad(\gamma<3)
$$

which is based on the experimental results of the ratio $v_{2} / v$ in the single-step shear. ${ }^{15}$

According to the BKZ constitutive model (or the Doi-Edwards model with IAA), the stress tensor $\boldsymbol{\sigma}\left(t, t_{1}, \gamma_{1}, \gamma_{2}\right)$ can be expressed in terms of relaxation stresses in single-step shear as

$$
\begin{gathered}
\boldsymbol{\sigma}\left(t, t_{1}, \gamma_{1}, \gamma_{2}\right)=\boldsymbol{\sigma}\left(t, \gamma_{2}\right)+\boldsymbol{\sigma}\left(t+t_{1}, \gamma_{1}+\gamma_{2}\right) \\
-\sigma\left(t+t_{1}, \gamma_{2}\right)
\end{gathered}
$$

where $\sigma$ stands for $\sigma, v$, and $v_{2}$. This equation gives proper prediction of $\boldsymbol{\sigma}\left(t, t_{1}, \gamma_{1}, \gamma_{2}\right)$ when $\gamma_{1} \gamma_{2}>0$ (i.e., $\gamma_{1}$ and $\gamma_{2}$ are parallel), but often fails when $\gamma_{1} \gamma_{2}<0$ as already mentioned.

On the other hand, the Doi-Edwards model without IAA gives the following equation as far as $t$, $t_{1} \geqq T_{\text {eq }}:^{7}$

$$
\begin{gathered}
\boldsymbol{\sigma}\left(t, t_{1}, \gamma_{1}, \gamma_{2}\right)=\boldsymbol{\sigma}\left(t, \gamma_{2}\right)+A(\beta)\left[\boldsymbol{\sigma}\left(t+t_{1}, \gamma_{1}+\gamma_{2}\right)\right. \\
\left.-\boldsymbol{\sigma}\left(t+t_{1}, \gamma_{2}\right)\right]
\end{gathered}
$$

where

$$
\begin{gathered}
A(\beta)= \begin{cases}(4 \beta / \pi)\left[\cos (\pi \beta / 2) /\left(1-\beta^{2}\right)\right] & (\beta<1) \\
(4 / \pi)\left[\cos (\pi / 2 \beta) /\left(1-\beta^{-2}\right)\right] & (\beta>1)\end{cases} \\
\beta=\left\{\left[3+\left(\gamma_{1}+\gamma_{2}\right)^{2}\right] /\left(3+\gamma_{1}{ }^{2}\right)\right\}^{1 / 2}
\end{gathered}
$$

The difference between eq 19 and 20 is practically neglibile when $\gamma_{1} \gamma_{2}>0$, and even vanishes when $\gamma_{1}=$ $-\gamma_{2} / 2$. In the latter case, $\beta$ and $A(\beta)$ happen to become unity, though $\gamma_{1} \gamma_{2}<0$. Apart from these cases, however, eq 19 and 20 give often significantly different predictions.

Such an example is shown in Figure 8, where the observed values of $\sigma$ and $v_{3}$ are shown by the circles, 


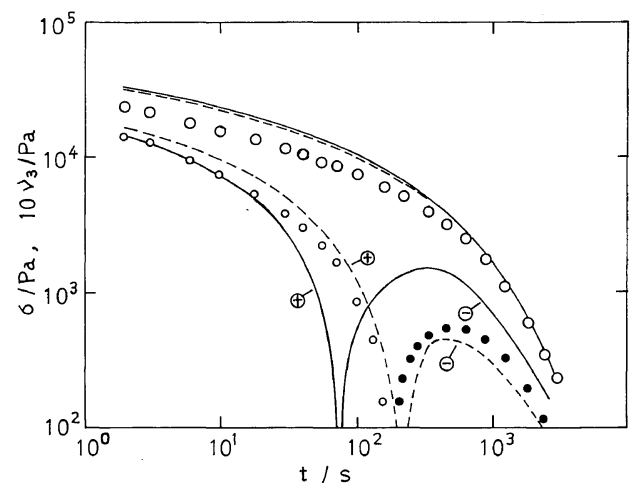

Figure 8. Shear stress $\sigma$ and normal stress difference $v_{3}=\sigma_{11}-\sigma_{33}$ in the relaxation process after application of a double-step strain which is specified by $t_{1}=200 \mathrm{~s}$, $i_{1}=-2.89$, and $i_{2}=1.45$. The solution used is the same as shown in Figure 6. Meaning of symbols and lines are given in the text.

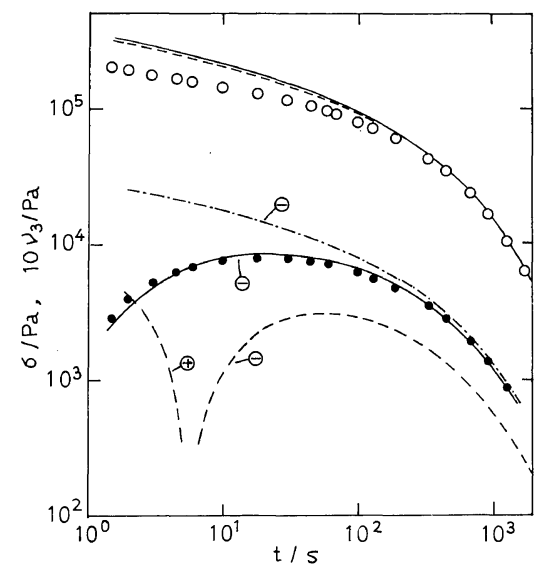

Figure 9. Relaxation stress components for the same type of double-step strain as that shown in Figure 8, but with $t_{1}=20 \mathrm{~s}$.

i.e., unfilled circles for positive sign of stresses and filled ones for negative sign, and the calculated values are shown by the solid lines (eq 19) and the dashed lines (eq 20), respectively. The polymer solution was the same as that shown in Figure 6, which was specified by the time constants, $\tau_{1}=T_{\mathrm{d}}=$ $1100 \mathrm{~s}, \tau_{k}=160 \mathrm{~s}, T_{\text {eq }} \cong 40 \mathrm{~s}$, and $\tau_{\mathrm{A}} \cong 0.1 \mathrm{~s}$. The strains adopted were $\gamma_{1}=-2 \gamma_{2}=2.89$, and the time interval $t_{1}$ was $200 \mathrm{~s}$, hence $t_{1}>\tau_{k}$. In this case, eq 20 gives a better account of shear stress than does eq 19, though a symptom of the opposite tendency is observed at several points in short times such that $t<20$ s.

The latter tendency becomes evident in Figure 9, where the time interval adopted is as short as $t_{1}=$ $20 \mathrm{~s}$. In this case, the observed values of $\sigma$ are in good agreement with the solid line due to eq 19 over the entire region of time examined. For $t \gg t_{1}$, the double-step shear becomes equivalent, in effect, to a single-step shear of $\gamma_{1}+\gamma_{2}$, leading to

$$
\sigma\left(t, t_{1}, \gamma_{1}, \gamma_{2}\right)=\sigma\left(t, \gamma_{1}+\gamma_{2}\right)
$$

This prediction shown by the dash-dotted line in Figure 9 is also in agreement with the experimental values for $t \gg t_{1}$. It is to be noted that eq 19 reduces to eq 23 for $t \gg t_{1}$ unless the total strain $\gamma_{1}+\gamma_{2}$ is too close to zero.

In this connection, it may be pertinent to reproduce here an explanation of the molecular process due to Osaki and Doi. ${ }^{18}$ Let us start with the state at $t<-t_{1}$, in which each primitive chain is in equilibrium and the attached Rouse segments are isotropic. This is schematically shown in (A) of Figure 10 , where the thin line represents the undeformed tube enclosing the right half of the chain and each circle represents a small group of isotropic segments. Of course, the center path of the tube is not really a straight line.

On application of the first negative strain $\gamma_{1}<0$ at $t=-t_{1}$, the tube is elongated and negatively oriented, together with the Rouse segments in it. This is shown in (B) by thick line and negatively inclined dashes. At around $t=-t_{1}+T_{\text {eq }}$, the chain returns to the equilibrium length in the deformed tube as shown in $(\mathrm{C})$, and a part of the deformed tube evaporates as represented by the dashed thick line. In the next period, a part of the primitive chain close to the end escapes from the deformed tube and recovers an equilibrium configuration in a renewed tube as shown in (D), where the renewed tube is represented by the thin line. Thus, just before the application of the second strain $\gamma_{2}$, the tube enclosing the primitive chain becomes to consist of two parts, i.e., the inner part deformed in negative direction by $\gamma_{1}$ and the outer part undeformed.

On application of the second strain $\gamma_{2}>0$, the outer part is stretched by a factor $\alpha=\left(3+\gamma_{2}{ }^{2}\right)^{1 / 2}$, while the inner part is deformed by a factor $\beta$ given in eq 22 , since it is already stretched by a factor $\left(3+\gamma_{1}^{2}\right)^{1 / 2}$. Thus, under the condition that $\gamma_{1}=$ -2.89 and $\gamma_{2}=1.45$, the inner part of the tube is really compressed since $\beta=0.67$, while the outer 
part is stretched by the factor $\alpha=2.25$. As a result, a considerable gap in the density of Rouse segments is induced between two parts of the tube at $t=0+$, as illustrated in $(\mathrm{E})$. The Rouse segments in the inner part are still negatively directed, while those in the outer part are positively directed.

If the gap in the segment density is equilibrated by the transfer of segments from inner to outer parts, the chain attains a state as shown in $(F)$ at the end of the equilibration process, i.e., at around $t=$ $T_{\text {eq }}$. In the process from (E) to (F), two negatively directed groups of segments slide into the positively directed tube and change to positively directed groups of segments. On the other hand, in the IAA model, the state at $t=T_{\text {eq }}$ is such that shown in $\left(\mathrm{F}^{\prime}\right)$.

Doi ${ }^{19}$ has recently developed a dynamics of the process $\mathrm{B}\left(\tau_{\mathrm{A}} \leqq t \leqq T_{\mathrm{eq}}\right)$ with the aid of IAA, and derived an expression for the relaxation stress in this process:

$$
\sigma(t, \boldsymbol{E})=3 \rho\left(N_{\mathrm{0}} / N_{\mathrm{e}}\right) k_{\mathrm{B}} T \mu_{\mathrm{B}}(t, E) Q_{12}(E)
$$

where $Q_{12}(\boldsymbol{E})$ is given by eq 9 and $\mu_{\mathbf{B}}(t, \boldsymbol{E})$ is given as

$$
\mu_{\mathrm{B}}(t, \boldsymbol{E})=\sum_{\mathbf{p}, \text { odd }} \frac{8}{p^{2} \pi}\left[1+[\alpha(\boldsymbol{E})-1] \exp \left(\frac{-t p^{2}}{T_{\text {eq }}}\right)\right]^{2}
$$

Thus, the relaxation stress $\sigma(t, \boldsymbol{E})$ is not separable into a function of time and that of strain. The corresponding expression for $\sigma(t, \boldsymbol{E})$ without the use of IAA has not been presented as yet.

\section{BIMODAL CHAIN ORIENTATION IN DOUBLE-STEP SHEAR}

As illustrated in Figure 10, there are two differently oriented segments in a polymer chain after application of double-step strain. Thus, we may extend eq 14 and $15 \mathrm{as}^{20}$

$$
\begin{aligned}
& 2 \sigma=\sigma_{\mathrm{P}} \sin 2 \chi=\sigma_{\mathrm{PI}} \sin 2 \chi_{\mathrm{I}}+\sigma_{\mathrm{PII}} \sin 2 \chi_{\mathrm{II}} \\
& \nu=\sigma_{\mathrm{P}} \cos 2 \chi=\sigma_{\mathrm{PI}} \cos 2 \chi_{\mathrm{I}}+\sigma_{\mathrm{PII}} \cos 2 \chi_{\mathrm{II}}
\end{aligned}
$$

with $2 \chi_{\mathrm{I}}=\cot ^{-1}\left[\left(\gamma_{1}+\gamma_{2}\right) / 2\right]$ and $2 \chi_{\mathrm{II}}=\cot ^{-1}\left(\gamma_{1} / 2\right)$.

If $\boldsymbol{V}$ is a vector of magnitude $\sigma_{\mathrm{P}}$ and orientation angle $2 \chi$, and $\boldsymbol{V}_{i}$ is a vector of $\sigma_{\mathrm{P} i}$ and $2 \chi_{i}(i=\mathrm{I}$ and II) in the $v, 2 \sigma$-plane shown in Figure 7, the above equations are simply expressed as

$$
\boldsymbol{V}=\boldsymbol{V}_{\mathrm{I}}+\boldsymbol{V}_{\mathrm{II}}
$$

The two sets of stress data shown in Figures 8 and

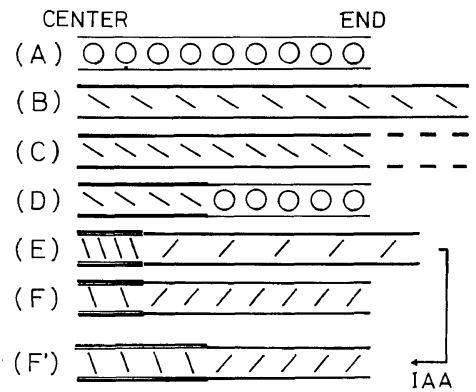

Figure 10. Change in the tube length, and in the orientation and density of Rouse segments at various stages of a double-step shear experiment.

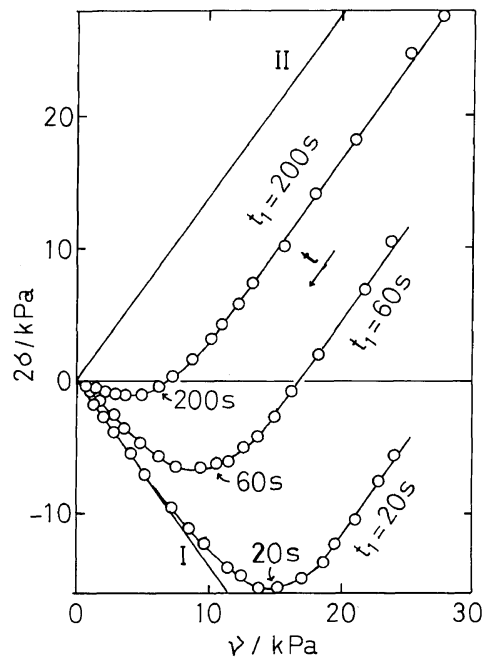

Figure 11. Two dimensional diagram of the stress state $(v, 2 \sigma)$ after application of double-step strain, in which $\gamma_{1}=-2.89, \gamma_{2}=1.45$, and $t_{1}=20,60$, and $200 \mathrm{~s}$, respectively.

9 , together with another set for $t_{1}=60 \mathrm{~s}$, are replotted by circles in Figure 11, where the lines I and II are drawn parallel with vectors $V_{\mathrm{I}}$ and $\boldsymbol{V}_{\mathrm{II}}$, respectively, and the data points for $t=t_{1}$ are indicated by arrows. In each case, the representative point moves parallel with $\boldsymbol{V}_{\text {II }}$ at the early stage of relaxation, indicating that only the outer part II of the chain is responsible for the relaxation at this stage. The relaxation of mode II finishes while the mode I starts at around $t=t_{1}$. This is to be expected, since the preparation and elimination of the outer part II are governed by the same kinetics whatever the mechanism. For example, the outer tube is 


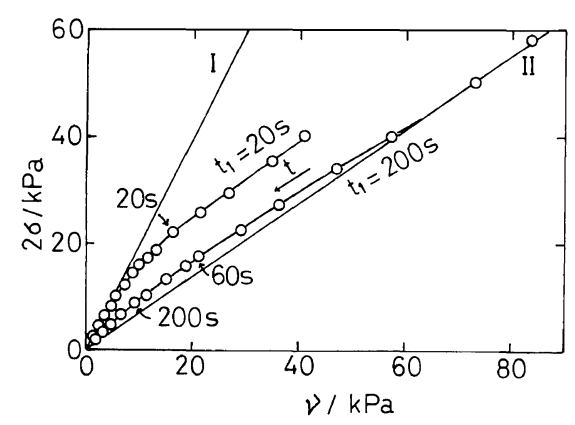

Figure 12. Two dimensional diagram of the stress state $(v, 2 \sigma)$ after application of double-step strain, in which $\gamma_{1}=-1.89, \gamma_{2}=2.88, t_{1}=20$ and $200 \mathrm{~s}$, respectively.

prepared and eliminated mainly by the reptation of chain when $t_{1}$ and $t>T_{\mathrm{eq}}$, and by the wriggling motion of segments when $t_{1}$ and $t<T_{\text {eq }}$.

The ideal separation of two modes occurs in the case of $t_{1}=20 \mathrm{~s}$. Here the outer tube is so short as compared with the inner tube that the latter long survives the former. On the other hand, in the case of $t_{1}=200 \mathrm{~s}$, the inner part is already too short for displaying the relaxation of pure mode I. In such a case, there must be a high probability that two modes proceed simultaneously in different molecules and even at different ends of the same molecule. The latter effect is, however, rather insignificant according to the Doi theory. ${ }^{7}$

Figure 12 shows the stress data for the same polymer system as that in Figure 11, but with another combination of strains that $\gamma_{1}=1.89$ and $\gamma_{2}=2.88 .{ }^{21}$ It is notable on the curve for $t_{1}=200 \mathrm{~s}$ that the contribution of inner segments $\sigma_{\mathrm{PI}}$ are enhanced in the range between 10 and $60 \mathrm{~s}$. Since $t_{1}>T_{\text {eq }}$ in this case, we obtain $\alpha=3.36$ and $\beta=0.78$ for the state $(E)$ in Figure 10. Then, the equilibration gives rise to an enhancement of the outerpart as in the case of the previous example. Of course, the scheme from (E) to $(F)$ should not be applied to a rapid process such that $t<T_{\text {eq }}$, even if $t_{1}>T_{\text {eq }}$. Thus, the problem may be tentatively interpreted in terms of eq 25. If this type of equation is applied to the inner part, the relaxation factor $\mu_{\mathrm{B}}(t, \boldsymbol{E})$ predicts an enhancement of $\sigma_{\mathrm{I}}$ since $\alpha_{\mathrm{I}}=\beta<1$. On the contrary, it predicts a larger decrease of $\sigma_{\text {II }}$ in the outer part, since $\alpha_{I I}=\alpha>1$. The corresponding analysis due to the slip-link model is anticipated.

Acknowledgement. I thank Dr. K. Osaki for many useful conversations. We acknowledge the financial support of the Grant-in-Aid for Scientific Research, 59470090.

\section{REFERENCES}

1. Y. Einaga, K. Osaki, M. Kurata, S. Kimura, and M. Tamura, Polym. J., 2, 550 (1971); See also, M. Tamura, M. Kurata, K. Osaki, Y. Einaga, and S. Kimura, Bull. Inst. Chem. Res., Kyoto Univ., 49, 43 (1971).

2. B. Bernstein, E. A. Kearsley, and L. J. Zapas, Trans. Soc. Rheol., 7, 391 (1963); 9, 27 (1965).

3. K. Osaki, S. Ohta, M. Fukuda, and M. Kurata, $J$. Polym. Sci., Polym. Phys. Ed., 14, 1701 (1976).

4. K. Osaki, "Proceedings of the 7th International Congress on Rheology," C. Klason and K. Kubát, Ed., Gothenburg, 1976, p 104.

5. M. Fukuda, K. Osaki, and M. Kurata, J. Soc. Rheol. Jpn. (Nihon Reoroji Gakkaishi), 2, 110 (1974).

6. M. Doi and S. F. Edwards, J. Chem. Soc., Faraday Trans. 2, 74, 1789, 1802, 1818 (1978); ibid., 75, 38 (1979).

7. M. Doi, J. Polym., Sci., Polym. Phys. Ed., 18, 1891 (1980).

8. K. Osaki, S. Kimura, and M. Kurata, J. Rheol., 25, 549 (1981).

9. K. Osaki, K. Nishizawa, and M. Kurata, Macromolecules, 15, 1068 (1982).

10. K. Osaki, S. Kimura, K. Nishizawa, and M. Kurata, Macromolecules, 14, 455 (1981).

11. P. G. de Gennes, J. Chem. Phys., 55, 572 (1971).

12. M. Doi, J. Polym. Sci., Polym. Phys. Ed., 18, 1005 (1980).

13. J. D. Ferry, "Viscoelastic Properties of Polymers," 3rd ed, Wiley, New York, 1980, p 251.

14. M. Doi, J. Polym. Sci., Polym. Phys. Ed., 21, 667 (1983).

15. K. Osaki, S. Kimura, and M. Kurata, J. Polym. Sci., Polym. Phys. Ed., 19, 517 (1981).

16. K. Osaki, N. Bessho, T. Kojimoto, and M. Kurata, J. Rheol., 23, 617 (1979).

17. S. Kimura, K. Osaki, and M. Kurata, J. Polym. Sci., Polym. Phys. Ed., 19, 151 (1981).

18. K. Osaki and M. Doi, Polym. Eng. Rev., 4, 36 (1984).

19. M. Doi, J. Polym. Sci., Polym. Phys. Ed., 18, 2055 (1980).

20. K. Osaki and M. Kurata, J. Polym. Sci., Polym. Phys. Ed., 20, 623 (1982).

21. S. Kimura, Dr. Eng. thesis, Kyoto Univ., 1982. 